\title{
$\mathrm{QR}$ 코드를 활용한 주제중심 통합학습 설계 사례연구
}

\author{
박형성 \\ 한국교원대학교 \\ hyungsung@gmail.com
}

\section{A Case Study on Design of Theme-based Integrated Learning by Using QR Code}

Hyung-Sung Park

Dept. of Educational Technology, Korea National University of Education

\begin{abstract}
요 약
본 연구의 목적은 스마트 기기를 활용한 교수-학습 설계와 학습방법에 대한 새로운 접근 및 방향을 제안하는데 있으며, 첨단 매체를 활용하여 학습자 참여를 촉진하는 게임형태의 주제중 심 통합학습 교수설계의 사례를 제시하였다. QR코드를 활용한 주제중심 통합학습의 사례인 퀘 스트 기반학습은 학습동기, 경험학습, 사회적 상호작용을 촉진하는 역할을 한다. QR코드를 활용 한 설계는 자신의 인지구조를 확장하는데 요구되는 사회적 상호작용 전략, 동기증진 전략, 구성 주의에서 강조하는 복잡한 문제 상황과 스캐폴딩 전략이 포함된 학습자 중심 학습 환경을 지원 하였다. 스마트 기기를 이용한 게임형 퀘스트 기반학습을 통해 학습에 대한 흥미 증진, 학습자 중심의 참여 학습, 활동중심, 협력학습, 사회적 상호작용을 통한 지식의 창출 측면에 대한 교수 설계에 창의적인 학습 환경을 제공하는 기회를 확인하였다.
\end{abstract}

\section{ABSTRACT}

This research aims at suggesting a case of designing theme-based integrated learning for usage smart media. New approach and direction for developing a gaming instructional design was suggested which can encourage learners to participate in. Quest-based learning, the learning environment design where learners conduct various learner-centered activities, plays an important role of reinforcing the motivation, promoting experiential and cooperative learning based on social interaction. The design using QR codes has been proved to be able to offer the learner-centered learning environment which includes social interaction strategy required for learners expanding their cognitive structure, motivation enhancing strategy encouraging their consistent participation in learning, complex problematic situation and scaffolding strategy emphasized by constructivism. And it is expected to contribute to promoting the design of theme-based integrated learning which is being demanded in the educational environment recently by combining systematic design process and strategies.

Keywords : QR code(QR코드), Game(게임), Quest-based learning(퀘스트 기반학습)

Received: Mar. 20, 2013 Revised: May 02, 2013

Accepted: May 15, 2013

Corresponding Author: Hyung-Sung Park

(Korea National University of Education)

E-mail: hyungsung@gmail.com

ISSN: 1598-4540 / elSSN: 2287-8211
(C) The Korea Game Society. All rights reserved. This is an open-access article distributed under the terms of the Creative Commons Attribution Non-Commercial License (http://creativecommons.otg/licenses/by-nc/3.0), which permits unrestricted non-commercial use, distribution, and reproduction in any medium, provided the original work is properly cited. 


\section{1. 서 론}

무선네트워크 기반의 스마트(smart) 기기를 활 용한 스마트러닝(smart-learning)이 학습자 중심의 학습활동 유형으로 자리 매김하고 있다. 정보통신 기술이 융합된 교수학습 환경에는 다양한 정보 유 형이 존재한다. 사용자들은 이러한 정보를 활용하 여 지식과 경험을 창출하는 활동을 한다. 기술이 융합된 학습 환경은 사용자들로 하여금 스마트 미 디어 환경에서 정보교환과 의사소통이 가능한 기회 를 제공한다.

매체가 통합된 구성주의 학습 환경은 학습자들 이 활동중심의 학습수행, 사고를 수반한 체계적인 경험, 다양한 유형의 상호작용, 이를 통한 반성과 성찰 활동을 통해 인지구조의 확장을 이끌어 나가 는 일련의 구조화된 경험의 기회를 제공한다. 학습 자들은 문제를 해결하는 능력의 근간이 되는 축척 된 경험을 통해서 확장된 경험을 창출하고 개인의 능력을 지속적으로 발전시켜 나가게 된다.

구성주의 교육은 학습자의 능동적인 자율성을 바탕으로 이루어지며, 학습자는 교수-학습 활동과 정에서 기존에 알고 있는 지식을 사용하거나 새로 운 지식을 창출하는 활동을 통해 자신의 배경지식 을 비슷한 방법으로 적용하고, 그 의미를 새겨봄으 로써 학습 과제와 관련된 내용을 이해하게 된다[1]. 이러한 과정에서 학습자 상호간, 학습자와 교사의 상호작용을 통해 자신의 의견이나 아이디어를 교환 하고, 학습내용에 대한 이해를 증진하며 교과에 대 한 학습자의 지식이나 생각을 재구조화 하게 된다. 인지구조의 확장은 개인이 속한 학습 환경과의 상 호작용을 통해 이루어지게 되며, 학습자들의 지식 구성은 개인이 속한 사회의 구성원들에 의해 영향 을 받는다. 지식을 구성하고 창출하는데 있어 나 이외의 사람들과 상호작용을 통해 인지구조를 확장 해 가며, 다른 사람들의 견해를 통해 자신이 구축 한 지식을 검증하고 확인한다.

학습자는 환경과 상호작용에서 얻는 경험의 재 구성 과정을 통해 지식을 구성한다. Duran 과
Gauvain[2]은 동료 간 상호작용이 문제해결을 위 한 단서를 제공한다고 주장하며, 학습자 자신의 방 식대로 학습 문제를 정의하고 재구조화하여, 문제 해결 전략을 수립하게 하여 학습을 촉진할 수 있 다고 하였다. 이처럼 사회적 상호작용을 통한 경험 의 확장은 스마트 미디어를 활용하여 시간과 공간 의 제약을 극복하고 활동중심의 팀 프로젝트 수업 이나 협동학습을 효율적으로 지원할 수 있다. 스마 트 미디어가 지원되는 주제중심 통합학습과 같은 최적화된 구성주의 학습 환경은 문제 상황에서 학 습자들이 문제해결을 위한 실마리나 단서로 활용할 수 있는 상황이나 맥락을 충분히 제공할 수 있는 기회가 되는 것이다.

교육현장에서 논의되는 주제중심 통합은 그동안 적용하였던 교과와 교과 간 통합이라는 통합방식을 넘어 학습자들의 활동을 다양한 주제중심으로 운영 을 하는 통합을 추구한다. 주제중심 활동을 위한 학습 환경의 창출을 위해서 사용자 참여, 공유, 사 회적 네트워킹 등이 적용된 스마트러닝 교수설계가 효율적이며, 이러한 기능을 충실히 지원하는 $\mathrm{QR}$ 코 드를 활용한 교수설계가 학습자 중심의 주제중심 통합 환경을 적절히 지원할 수 있다. 김선진[3]은 이를 통해 교육 콘텐츠(content), 교수자와 학습자 사이의 자연스럽고 생산적인 상호작용이 증진되고 학습자의 학습에 대한 몰입(flow)이 증진될 수 있 다고 주장하였다.

QR코드(Quick Response Code)를 활용한 게임 형 퀘스트 기반학습(quest based-learning)은 학습 활동에서 주어지는 일련의 문제 상황 속에서 학습 자들이 학습목표 해결을 위한 다각도의 시도로 학 습과제를 해결하고, 이러한 과정에서 주어지는 연 습과 즉각적인 피드백을 통해 학습목표를 해결하는 과정이 반복적으로 일어난다. 상위 학습목표를 해 결하기 위해 안내된 학습과제를 수행하며 실수를 통한 자기자각 효과를 얻을 수 있으며 성공했을 때 보상으로 강화를 제공 받을 수 있다. 또한 문제 상황에 대응하기 위해 학습자 스스로 자신의 주변 여건, 환경 등을 재구성하는 구성주의 학습 환경을 
창출 할 수 있다[4].

본 연구의 목적은 스마트(smart) 매체를 통합한 교수-학습 설계와 학습방법에 대한 새로운 접근과 방향 제시에 있다. 새로운 교수학습 방법이 효과적 인 학습목표 달성에 어떠한 가능성을 제공하고, 스 마트 기기를 이용한 게임형 퀘스트기반 학습을 통 해 학습에 대한 흥미 증진, 학습자 중심의 참여 학 습, 활동중심, 협력학습, 사회적 상호작용을 통한 지식의 창출 측면에 대한 교수설계 사례를 통해 그 가능성과 의미를 찾고자 한다.

\section{2. 이론적 논의}

\section{1 학습자 중심의 게임형 퀘스트 학습}

교육자들은 첨단학습기술을 교육 상황에 효과적 으로 활용하여 교육목표를 달성하고자 다양한 교수 설계를 시도해왔다. 정보통신기술과 관련된 초기 연구에서는 주로 이러한 기술의 사용을 가르치는 소양교육에 초점을 두었지만 기술 자체로는 교수학 습 상황에서 교육목표를 효과적으로 달성하는데 활 용되기는 어려운 문제가 대두되었다.

학습활동의 핵심은 학습자들이 구성하는 지식, 경험의 양과 질, 이를 통한 인지구조의 확장에 있 다. 매체에 대한 소양을 넘어 적시 적절한 활용을 통해 학습자들이 스스로 얻게 되는 의미의 창출, 인지구조와의 효과적인 통합이 중요한 것이다.

학습자들은 스마트 기기를 활용하여 문제를 해 결하는 과정에서 다양한 방법을 통해 시행착오 과 정을 거치며 학습에 임한다. 정보를 검색하고, 수 집하며 자신의 전략으로 정보를 정교화 하는 과정 을 통해 자신에게 적절한 학습전략들을 활용하여 문제를 해결한다. 이러한 학습전략들은 학습자가 학습 중에 정보나 지식 및 기술을 획득, 저장, 활 용을 촉진할 수 있는 일련의 과정과 단계, 즉 학습 과정 및 사고과정과 밀접한 관련을 지닌다. 학습자 의 학습과정 및 사고과정에서 어떤 전략을 지원하 여 사고할 수 있도록 하느냐에 따라 학습목표 달
성의 효율성도 증진될 수 있다.

퀘스트 기반학습(Quest-based Learning)은 학 습활동에서 주어지는 일련의 문제 상황 속에서 학 습자들이 학습목표(quest)를 해결하기 위해 이야기 (story: 학습과제)에서 주어진 인물, 배경, 사건을 통해 문제를 확인하고, 해결하기 위한 계획을 수립 하며 다양한 시도를 통해 문제를 해결하고, 이러한 과정에서 연습과 즉각적인 피드백, 상호작용을 통 해 학습목표를 해결하는 과정이 반복적으로 일어나 도록 하는 학습이다. 이러한 학습 과정에서 $\mathrm{QR}$ 코 드를 접목한 교수설계는 다양한 상호작용 활동이 가미되어 학습자들의 동기[4], 몰입, 학습 전이 등 에 중요한 의미를 지닌다.

교수-학습 활동 과정에서 학습자들의 학습활동 에 영향을 미치는 상호작용은 학습자 인지구조의 확장과 정서적, 육체적 기술의 발달을 촉진하는 필 수 요소이며, 새로운 미디어를 교수학습에 통합하 여 학습자 중심의 학습과정을 설계하는데 있어서 공유와 협력, 의사소통을 위한 중요한 역할을 한다.

상호작용은 컴퓨터와 같은 매체를 사용하는 정 보화 시대에서 중요한 요인임에 틀림없으며 교수학 습 상황에서 학습자 중심의 의미 있는 학습 결과 를 만드는데 있어서 유기적인 역할을 한다[5].

박형성[4]은 퀘스트 기반학습에서 주어지는 문제 (quest)는 학습자가 해결해야 하는 학습목표와 학 습과제로 볼 수 있다. 퀘스트는 게임에서 임무 (mission)로 제시되고, 게임의 진행흐름과 자연스 럽게 연결 되어야 하며 게임의 몰입을 방해하지 않도록 계획되어야 한다고 이야기한다. 또한, 교육 현장에서 요구하는 요구를 달성할 수 있도록 학습 자에게 친근하게 제공되어야 하며, 임무가 어려워 접근성이 약하거나, 몰입도가 떨어지지 않도록 고 려해야 한다고 밝히고 있다.

퀘스트 기반학습에서 학습자가 학습과제를 의미 있게 수용하기 위해서는 학습 초반부터 학습목표의 필요성과 취지를 분명히 이해할 수 있도록 밝혀야 한다. $\mathrm{QR}$ 코드를 통한 설계에서 초기 임무 제공시, 텍스트 메시지보다는 문제 상황을 충분히 인식할 
수 있는 영상을 통해 제공하는 것이 좋은 대안이 될 수 있다. 이는 수업을 실제 상황과 관련시킴으 로써 내용의 유의미성을 증진시키는 중요한 역할을 하기 때문이다. 학습과제도 단순화시키기보다 복잡 한 상황을 어느 정도 노출시켜서 그러한 문제 상 황에서 문제를 해결하기 위한 다양한 방법을 활용 할 기회를 줄 필요가 있다는 것이다. 게임형 퀘스 트 기반학습은 학습자에게 일반적인 지식을 단순히 전달하는 수준의 수업보다 학습자가 능동적으로 자 신의 인지능력을 발휘할 수 있는 학습 환경을 제

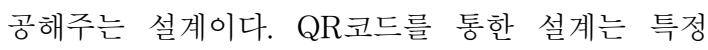
상황에 대한 해석과 예측, 가설을 만들어 탐구하는 사고력, 자신의 생각과 전략과 기법을 충분히 발현 해볼 수 있는 학습활동이 설계될 수 있다.

활동과정에서 학습자들은 $\mathrm{QR}$ 코드로 설계된 임 무를 달성하기 위해 팀별로 협동학습 활동을 수행 한다. 이때 설계에 반영된 게임이 지니는 특성인 경쟁, 과제, 탐험, 환상, 목표, 상호작용, 규칙, 강화 나 보상 등을 주기적으로 경험하게 된다. $\mathrm{QR}$ 코드 에서 제공되는 임무에 포함된 규칙, 팀원과의 상호 작용을 통한 문제해결, 설계된 11 개의 임무에서 난 이도가 조절되어 제공되는 학습과제, 11 개의 임무 를 해결하기 위해 임무가 제시된 곳을 이동하고 해결하는 탐험활동과 노력하는 태도, 11 개의 임무 가 하나의 스토리로 이뤄져 탐험과정에서 환상을 경험하게 된다. 또한 $\mathrm{SNS}$ 와 연동되어 실시간 각 팀별 임무의 달성과 진행과정을 확인함으로써 팀별 강화나 보상을 획득하고 11 개의 미션을 달성했을 때 상위 팀에게 보상이 제공된다.

이러한 활동과정에서 협동학습은 참여자의 다 관점과 의견을 반추하여 자신의 입장을 규명하고, 조율하면서 일치된 의견을 이뤄가는 학습 방법으 로, 팀원들 간의 상호교류와 반성적 사고, 생성적 학습을 촉진한다. 협동학습을 통한 상호작용은 동 료간 신뢰, 서로에 대한 적극적인 태도, 다른 사람 에 대한 책임감, 타인에 대한 존경심을 가져온다. 협동학습에서는 모든 집단구성원들이 그 집단의 학 습목표를 달성하는 데 기여하기 때문에 구성원 모
두가 성공 경험을 갖게 됨으로써 학습태도 및 학 습동기의 유발에 기여한다.

$\mathrm{QR}$ 코드를 활용한 퀘스트 부여와 이를 통한 상 호작용은 근접발달영역에서 학습자들에게 스캐폴딩 (scaffolding)로서 역할을 수행할 수 있다. 이는 학 습자 스스로 할 수 있는 능력이 부족하지만, 팀원 들 각자 역할에 따른 협동과 수행능력에 따라 협 력자, $\mathrm{QR}$ 코드 퀘스트를 통해 도움을 주거나 스캐 폴딩 역할을 수행한다면, 학습자 스스로 할 수 있 는 능력이 증가하게 되고, 궁극적으로 과제를 해결 할 수 있는 능력을 습득할 수 있다.

퀘스트 기반학습의 성공은 임무들이 얼마나 게 임의 특성을 반영하여 설계되고, 학습자에게 재미 와 흥미를 유발할 수 있느냐가 관건이다. 동기를 증가시킬 수 있는 방법은 학습자에게 도전정신을 유발시킬 수 있는 학습과제의 구조화, 퀘스트 단계 별 난이도를 조절하여 성취감을 맛보게 할 수 있 어야 한다. 또한 이야기 구조, 상호작용, 임무, 학 습결과, 학습상황의 요소들이 학습자가 게임을 진 행하면서 단순한 경험이 아닌 학습목표 달성을 위 한 과정의 일부인 '성찰적 경험'이라는 새로운 배움 을 얻을 수 있도록 계획되어야 한다.

$\mathrm{QR}$ 코드를 이용한 학습에서 학습자들은 활동적 이고 인지적인 경험을 통해 팀원들 간의 강한 유 대감과 존재감을 경험하며, 학습활동 과정에 적극 적으로 참여할 수 있다. 인간은 유기체, 기계등과 같이 자신 이외의 환경에 존재하는 다양한 객체들 과 상호작용을 하려는 본성을 지니고 있으며, 정보 화 사회의 원천 중 하나인 컴퓨터와 애착을 맺는 존재이며[6], 기존의 연구에서도 상호작용성이 높은 교수매체가 학습자의 자기주도적 학습에 긍정적인 영향을 미친다는 연구결과를 내놓고 있다[7].

\section{2 주제중심 통합학습}

통합학습은 학습자의 경험, 인지능력, 흥미, 요구 등을 반영하며, 학습자들이 문제 상황에서 주어진 단서들을 구조적이고 조직적으로 결합하여 문제해 결에 활용함으로써 학습의 폭을 넓히도록 하는 방 
법이다. 통합 주제선정은 교과서나 교육과정상의 주제, 학생들의 인지 능력에 맞는 쟁점과 관심사, 인류의 과제에 해당하는 주제를 폭넓게 다루게 된 다[8]. 주제중심 통합학습은 교과별 내용을 엄격히 분할하여 가르치는 방식이 지닌 한계의 대안적 접 근으로 교과 영역의 경계를 무너뜨려 융합형태로 문제나 쟁점, 사고기능, 개념, 범주 등을 중심으로 여러 교과 등의 학습 내용을 통합적으로 재조직하 여 가르치거나 학습하도록 하는 방식이다[9].

주제중심 통합학습은 학습자의 요구, 흥미에 부 합하고 사회가 요구하는 문제해결능력과 창의력 개 발을 위한 기회를 제공한다. Spanos[10]는 학습자 들이 흥미를 느끼는 주제를 선정하는 것이 중요함 을 강조하였으며, Jacobs[11]는 중심 주제를 고찰 함에 있어 하나 이상의 과목에서 언어와 수업방법 을 적용하는 것이라고 하였다. 주제중심 통합학습 은 학습자들이 경험하는 다양한 문제 해결을 위해 개인이 지닌 지식과 경험을 종합하여 응용할 수 있는 통합적 이해와 활용이 중요한 역할을 한다.

Ackerman[12]은 주제중심 통합학습의 접근 지 침을 학습자들이 찾아낸 개념들이 다른 교과에 단 순히 관련되기만 한 것이 아니라 해당 교과에서도 중요한 것인지 확인해야 하며, 교과개념에 대한 학 습을 실질적으로 높여 주는 것인지, 단원(unit)은 구성하고 있는 다른 교과의 단원을 통합하거나 초 월하는 감각을 개발시키는 의미가 있는지 확인하 며, 단원이 바람직한 지적 성향을 개발할 수 있어 야 한다고 하였다. QR코드를 활용한 퀘스트 기반 학습은 범교과적 주제를 통합하여 교수학습을 진행 할 수 있는 환경을 제공하며, 주제중심 통합학습을 위한 도구의 역할 하는데 충분한 의미를 지닌다.

\section{3. 설계 사례연구}

\subsection{Eco Project 개요}

$\mathrm{QR}$ 코드를 활용한 주제중심 통합학습인 [Eco!! 비상대책위원회]는 환경, 기술, 과학 교육이 융합된
주제이다. 퀘스트 기반학습 설계 사례에 대한 구체 적인 개요는 다음과 같다.

목표는 학생들이 에너지 문제를 해결하기 위해 다양한 자료를 수집하는 과정에서 실제적인 문제를 해결하고, 활동과정에서 친환경 주택에 대한 학습 내용을 이해하고, 창의적 능력, 문제해결력, 의사소 통, 협력, 테크놀로지 리터러시, 도전의식 등을 함 양하는데 있다.

학습 방법은 퀘스트 기반학습과 프로젝트기반학 습으로, 환경과 에너지문제를 해결하기 위하여 자 료수집, 웹 포스터를 제작하여 홍보하는 학습활동 을 수행하게 된다.

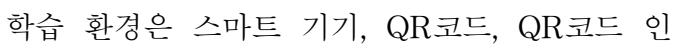
식 앱(App), 페이스북과 같은 $\mathrm{SNS}$, 글로그스터 등 을 연동하여 사용한다.

\section{2 설계 절차}

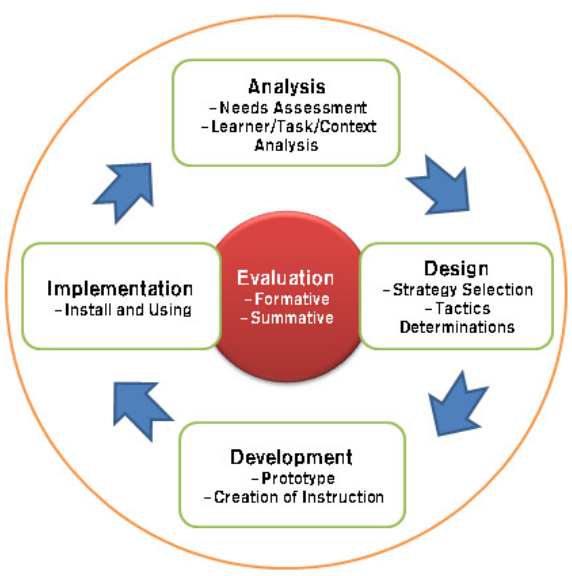

[Fig. 1] Instructional design procedures

\section{3 분 석}

\subsection{1 요구분석}

요구분석은 교수학습활동의 대상이 되는 $\mathrm{C}$ 도 소 재 중학교 3학년 학생(나이 평균 16세) 150명을 대상으로 초점집단 면접 30명, 설문조사 150 명(초 점집단 면접 포함)을 대상으로 실하였다. 요구분석 
을 위해 개방형 질문지를 토대로 면접을 진행하였 다. 요구분석의 주요 내용은 최적화된 학습 환경으 로서 스마트 기기가 도입된 학습자 중심의 학습 환경에 대한 사용의도, 교수학습 프로그램에서 요 구되는 기능과 새로운 수업방법에 대한 인식과 방 향, 경험과정에서 학습자들이 얻고자 하는 지식과 기능을 중심으로 이루어졌다.

(1) 최적: 친환경 건설기술과 관련하여 실제적인

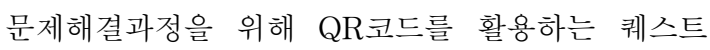
기반학습의 개발이 요구되며, 교육과정, 평가, 방법 등에서 21세기 학습자 역량이 반영될 필요가 있다.

(2) 실제: 학습자들은 $\mathrm{QR}$ 코드를 활용한 퀘스트 기반학습에 대한 경험이 없으며, 강의식 수업 위주 로 운영되어 활동중심 및 구성주의적 학습방법에 대한 경험이 부족하여, 친환경건설기술 관련 문제 를 해결하는 과정에서 $\mathrm{QR}$ 코드는 문제해결과정과 관련된 임무 및 단서 제공, 임무 수행과정에서 필 요시 교사 1 인의 스캐폴딩을 제공할 수 있다.

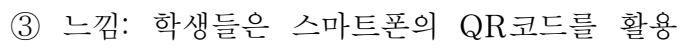
한 퀘스트 기반학습을 접하지 않아 생소함과 활동 을 통한 학습에 대한 호기심 가지고 있다.

(4) 해결방안: 친환경 건설기술과 관련해 실제적 인 문제해결 과정을 통한 학습이 중요하며, $\mathrm{QR}$ 코 드를 활용한 퀘스트 기반학습을 통해 해당 문제를 자연스럽게 이해하고, 학습에 대한 흥미, 학습자중 심의 학습, 협동학습, 21 세기 학습자 역량을 고려 한 교수설계를 통해 해결할 수 있다.

\subsection{2 학습자 분석}

해당 교수설계 사례를 통해 학습하게 될 학습대 상의 일반적인 특성은 중학교 3학년 학생으로 평 평균 연령은 15-16세이다. 해당 연령의 학습자들의 인지적 특성은 피아제의 인지적 발달 단계를 근거 로 형식적 조작기로 명제적 사고(현실상황을 고려 하지 않고도 언어적 진술에 이한 명제의 논리를 평가)와 결합적 분석(명제적 사고와 문제해결과정 에서 관련 변인들을 추출, 분석, 상호 관련짓고 통
합), 추상적 추론 등을 활용하여 지식을 습득하는 특성이 있다.

정서적 특성은 학습자들은 여러 가지 매체를 통 해 친환경 기술과 신재생에너지의 필요성에 대해 흥미를 가지고 있었으며, 상급학교의 진학을 위해 성적과 관련된 곳에 집중하는 외적 동기를 지니고 있으며, 자신의 진로와 적성을 고려하기 위하여 실 제적인 과목을 배우기 바라는 내재적 동기를 가지 고 있다. 과목에 대한 태도는 기술교과의 실제적인 지식 활용의 중요성에 대해 인지하고 있으나 대부 분은 기술교과목이 어렵다고 인식하고 있었으며 $\mathrm{QR}$ 코드를 활용한 수업에서 교사의 보조와 안내를 받기를 희망하였다. 또한 기존에 교실에서만 이루 어진 학습에 익숙해져 있어 현장에서의 실제적인 문제해결 상황에 부딪혔을 경우 불안감을 갖는 불 안감 수준이 높은 것으로 나타났다.

\subsection{3 학습과제 분석}

학습주제는 “친환경 건설 기술의 이해 및 적용" 이며 중학교 기술교육과정 분석을 통해 추출한 학 습활동은 기술세계 영역에 해당하는 『건설 기술』 로 건설기술의 이해, 건설 구조물의 이용, 건설 구 조물 모형 만들기에 대한 학습과제가 주를 이루고 있으며, David Merrill의 학습과제 분석을 근거로 수행과 내용수준을 통해 미시적 관점에서 과제분석 을 [Table 1]과 같이 수행하였다.

[Table 1] Analysis of Learning Task

Micro Analysis(content X performance)

1. Definition of Active Technology

Technology understanding[concept $\mathrm{X}$ remember]

Technology case searching[fact $\mathrm{X}$ use]

Condition understanding[fact $\mathrm{X}$ find]

2. Application of Active Technology

Understanding of Photovoltaic power generation [concept X remember]

Difference of between sunlight and solar heat [concept X remember]

Concept of incidence angle[concept $\mathrm{X}$ remember] 
Concept of reflector[concept $\mathrm{X}$ remember]

Electric power station site[concept X use]

Electric power site case[concept X use]

Writing reason for selection of electric power site[concept $\mathrm{X}$ find]

\section{Definition of Passive Technology}

Technology understanding[concept $\mathrm{X}$ remember]

Technology case searching[fact $\mathrm{X}$ use]

Condition understanding[fact $\mathrm{X}$ find]

4. Application of Passive Technology

Definition of adiabatic[concept $\mathrm{X}$ remember]

Adiabatic case[concept $\mathrm{X}$ use]

Important of adiabatic[concept $\mathrm{X}$ find]

Way for adiabatic[principle $\mathrm{X}$ use]

Waste of electric power[concept X remember]

Case of waste of electric power[fact $\mathrm{X}$ use]

Important of power save[fact $X$ find]

Definition of rooftop greening[concept $\mathrm{X}$ remember]

Rooftop greening case[fact X use]

Selection site for rooftop greening[fact $\mathrm{X}$ use]

Important of rooftop greening[fact $\mathrm{X}$ find]

Important of windows direction[concept $\mathrm{X}$ remember]

Comparison of energy conservation among the south and north, east and west[fact $X$ find]

\section{4 설 계}

\subsection{1 수행목표}

학습과제 분석을 근간으로 수행목표를 설정하였 다. 수행목표는 학습자, 행동, 조건, 준거가 드러날 수 있도록 계획하였다.

- 친환경 건설 기술의 개념과 액티브 기술과 패 시브 기술을 적용한 사례를 말할 수 있다.

- 패시브와 액티브 기술을 실제 상황에 적용할 수 있는 방안을 말할 수 있다.

- 단열이 잘못된 사례와 낭비전력 사례를 찾아 말할 수 있다.

- 옥상녹화에 적합한 장소를 선정하고, 창호 방 향의 중요성을 설명할 수 있다.

- 친환경 건설기술 성찰일지를 작성할 수 있다.

\subsection{2 교수설계 전략}

교수설계전략은 교수과정전략, 동기증진전략, 상 호작용 전략으로, 학습자들이 학습과정에 몰입하여 학습목표를 효과적으로 달성하도록 조직하였다.

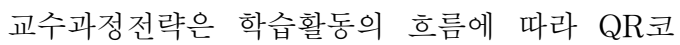
드 설계 시 학습자의 학습참여 의지를 증진하기 위한 전략들을 [Table 2]에 포함하였다.

[Table 2] Instructional Process Strategy

\begin{tabular}{|c|c|}
\hline Activity & Strategies \\
\hline Motivation & \multirow{5}{*}{$\begin{array}{l}\text { - Providing mission via } \\
\text { multimedia } \\
\text { - Providing learning } \\
\text { objective via QR code } \\
\text { - Providing hint for recall } \\
\text { via QR code }\end{array}$} \\
\hline Learning & \\
\hline & \\
\hline $\begin{array}{l}\text { Stımulate of } \\
\text { prior knowledge }\end{array}$ & \\
\hline $\begin{array}{c}\text { Guidance for } \\
\text { Learning }\end{array}$ & \\
\hline Resource Search & \multirow{3}{*}{$\begin{array}{c}\text { - Resource search with } \\
\text { Smart phone } \\
\text { - Information sharing, } \\
\text { feedback, and Interaction } \\
\text { via SNS } \\
\text { - Collaborative learning for } \\
\text { resolving the mission } \\
\text { - Scaffolding be considered } \\
\text { learner level }\end{array}$} \\
\hline $\begin{array}{l}\text { Collaborative } \\
\text { Learning }\end{array}$ & \\
\hline $\begin{array}{l}\text { Learning Task } \\
\text { Resolution }\end{array}$ & \\
\hline $\begin{array}{l}\text { Outcome making } \\
\text { / Presentation }\end{array}$ & \multirow{3}{*}{$\begin{array}{c}\text { - Poster making with } \\
\text { Glogster } \\
\text { - Reflection paper } \\
\text { Expert feedback } \\
\text { - self-appraisal } \\
\text { Peer Review }\end{array}$} \\
\hline Reflection & \\
\hline Assessment & \\
\hline & $\begin{array}{l}\text { Ig via SNS } \\
\text { Communication }\end{array}$ \\
\hline Teacher & $\begin{array}{c}\text { Co-Worker } \\
\text { Group }\end{array}$ \\
\hline
\end{tabular}

동기증진전략은 학습에 흥미와 관심을 일으키고 학습 동기를 유발할 수 있도록 하기 위해 Keller의 $\mathrm{ARCS}$ 모델을 활용하여 설계에 반영하였다.

주의집중(Attention) 전략으로, 첫 번째 임무에 서 영상매체를 통해 임무를 부여하고, 학습자 참여 
를 유도 하는 게임형 이벤트와 임무를 제공하고, 임무를 탐색하는 과정에서 문제 상황을 제시하면서 필요한 지식을 부분적으로 단서형태로 제공한다. 또한 다양한 상호작용을 위한 이벤트를 임무 중간 중간에 제시하여 주의를 집중하도록 한다.

관련성(Relevance)증진 전략으로, 학습자들이 $\mathrm{QR}$ 코드를 통해 쉽게 접하고 이해하며, 단서를 제 공받을 수 있는 문제 상황을 제시하며, 학습자가 주로 사용하는 구어체를 사용하여 시나리오를 전개 하고, 실제적 문제와 학습자들을 상황에 몰입하게 하여 해결 가능한 학습 목표(임무)를 제시하였으며, 전문가와 동료 학습자들 간의 피드백과 상호작용이 지원되도록 $\mathrm{SNS}$ 와 연동하여 주기적인 스캐폴딩을 할 수 있도록 하였다.

자신감(Confidence) 증진 전략으로, 학습목표를 명확하게 제시하고, 임무별 팀원들의 협력활동이 지원되도록 하였으며, 학습을 수료할 수 있는 조건 을 제시하여 통과할 수 있도록 하였다.

만족감(Satisfaction) 증진 전략으로, 학습자의 행동유지를 위한 긍정적인 피드백과 보상을 $\mathrm{SNS}$ 를 통해 지속적으로 제공하고, 경쟁을 통한 임무 달성정도에 따라 순위에 의해 강화를 하였다.

상호작용(interaction)은 학습자가 과제를 수행하 는데 있어 방향을 잃지 않게 하고, 학습의 과정을 유지시켜 주는 역할을 한다. 또 활동 과정을 성찰 하여 문제 해결 과정에 대한 이해력을 높이는 메 커니즘(mechanism)을 제공하는 중요한 요인이다 [5]. 상호작용이 원할 하게 이루어 질 수 있도록 3 가지 유형의 상호작용 증진 전략을 도입하였다.

첫째, 학습자간 상호작용이다. 학습자들은 인터 뷰 동영상, 사진 자료 등을 $\mathrm{SNS}$ 에 올려 실시간으 로 공유하고 댓글과 좋아요 등을 통해 의사소통 촉진하며, 학습자가 명확하게 임무를 수행할 수 있 도록 역할 분담이 이루어진다.

학습자간 상호작용을 위해 부여된 역할을 살펴 보면, 기관장은 팀원의 역할을 분담하고 협동을 촉 진하며, 도서관장은 팀원의 의견을 모아 글을 구성 한다. 홍보과장은 팀원이 수집한 자료를 바탕으로
홍보포스터를 제작하며, 시설과장은 지도를 보며 팀원의 방향을 안내하고 다음 미션 장소를 안내한 다. 학생회장은 해당 미션 장소에서 정보를 수집하 며, 학생부장은 문제해결을 위해 인터넷을 이용하 여 정보를 수집한다.

둘째, 학습자와 학습과제의 상호작용이다. 학습 자들은 학교의 구성원이 되어 역할을 실제 수행해 봄으로서 역할과 임무에 내재된 학습과제를 경험한 다. 이때 게임형태로 설계된 학습활동 절차가 학습 자의 흥미를 불러일으키고, 역할에 대한 몰입을 유

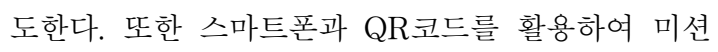
을 제시함으로써 흥미를 유발할 수 있으며, 최종 산출물을 포스터로 제작하여 학습에 대해 성찰 활 동을 진행할 수 있다.

마지막으로 학습자와 교수자의 상호작용이다. 학 습자가 미션을 수행하고 사회적 네트워크 서비스 (SNS)에 글을 올리면 교사는 피드백 제공 및 다 음 미션을 안내한다. 또한 제작된 포스터에 피드백 을 제공하고 수정 보완할 기회를 부여한다.

\section{5 개 발}

\subsection{1 문제 상황}

우리는 에너지 위기에 봉착하고 있습니다. 현재 의 에너지소비가 지속된다면 2015년 우리나라는 심각한 에너지 부족을 겪을 것이라 예상됩니다. 전 국의 학교에서도 에너지를 수급하기 위한 비용이 예산을 크게 초과하고, 각 기관의 에너지 절약과 생산에 대한 노력이 요구되고 있습니다. 이에 학교 에서 에너지가 낭비되고 있는 곳을 찾아 개선하고, 친환경 에너지를 생산하여 이용하는 역할모델이 되 어 주시기 바랍니다. 자! 지금부터 학교장은 $\mathrm{ECO}$ 비상대책위원회를 가동하여 학교에서 낭비되고 있 는 에너지를 줄이고, 친환경 에너지를 생산할 방법 을 찾아 친환경 학교를 만들어 주시기 바랍니다. 또한 다른 기관의 좋은 사례를 홍보할 수 있는 포 스터를 제작하여 홍보를 부탁드립니다. 


\subsection{2 이야기(story)}

학습자는 친환경 학교 홍보포스터를 제작하기 위하여 패시브와 액티브 기술 자료를 수집한다. 임 무 사이에 동기증진을 위한 게임형 임무가 있어 목표를 달성하게 되면 관련 자료를 1 개를 받을 수 있다. 수집된 자료를 바탕으로 포스터가 제작되며, 평가가 이루어진다. 아래는 연구 사례에서 제시한 11 개의 임무로 이야기 전개에 따라 게임형 임무 3 개, 학습형 임무 8개로 구성되어있다.

[Table 3] QR code mission

\begin{tabular}{|c|c|c|c|c|}
\hline M 1 & \multirow[b]{3}{*}{-} & M 2 & & M 3 \\
\hline Learning & & Learning & \multirow[b]{2}{*}{$\rightarrow$} & Game \\
\hline $\begin{array}{l}\text { Suggestion } \\
\text { of mission } \\
\& \text { situation }\end{array}$ & & $\begin{array}{l}\text { Interceptio } \\
\mathrm{n} \text { of heat }\end{array}$ & & $\begin{array}{l}\text { ECO words } \\
\text { completion }\end{array}$ \\
\hline M 4 & & M 5 & \multirow[b]{3}{*}{$\rightarrow$} & M 6 \\
\hline Learning & & Learning & & Game \\
\hline $\begin{array}{l}\text { Standby } \\
\text { power } \\
\text { reduction }\end{array}$ & & $\begin{array}{l}\text { Rooftop } \\
\text { greening }\end{array}$ & & Levitation \\
\hline M 7 & \multirow[b]{3}{*}{$\rightarrow$} & M 8 & \multirow[b]{3}{*}{$\rightarrow$} & M 9 \\
\hline Learning & & Game & & Learning \\
\hline $\begin{array}{c}\text { Energy } \\
\text { saving } \\
\text { pentastich } \\
\text { composing }\end{array}$ & & $\begin{array}{l}\text { Speed } \\
\text { game }\end{array}$ & & $\begin{array}{c}\text { solar } \\
\text { battery } \\
\text { locational } \\
\text { conditions }\end{array}$ \\
\hline M 10 & & M 11 & \multirow{2}{*}{\multicolumn{2}{|c|}{ Evaluation }} \\
\hline Learning & & Learning & & \\
\hline $\begin{array}{l}\text { Using of } \\
\text { solar } \\
\text { energy }\end{array}$ & $\longrightarrow$ & $\begin{array}{l}\text { Promoting } \\
\text { poster } \\
\text { making }\end{array}$ & $\rightarrow$ & $\begin{array}{c}\text { Poster } \\
\text { presentation }\end{array}$ \\
\hline
\end{tabular}

\subsubsection{QR코드 퀘스트 개발 사례}

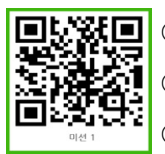

○ 제목: 임무부여/상황제시

○장소: 본부건물

미션1: 여러분은 에너지 절약을 위 한 비상대책 위원들 입니다. 동영상을 보고 문제를 확인하기 바랍니다. 모둠원간의 회의를 통해 역할 을 맡으시기 바라며, 미션을 해결하여 친환경 학교
를 만들기 바랍니다.

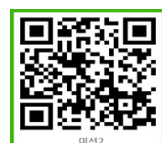

제목: 이중창으로 열 차단 ○장소: 인문과학관

○미션2: 인문과학관에서 에너지가 낭 비되고 있다는 신고가 들어왔습니다. 에너지가 새 고 있는 창문을 1 개 찾아 사진을 찍어 facebook에 올리세요.

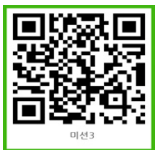

O 제목: $\mathrm{ECO}$ 글자 완성하기 $\bigcirc$ 장소: 제한 없음

미션3: 보다 많은 사람들에게 에너지 절약을 홍보하기 위해 주변 사물 중 $\mathrm{E}, \mathrm{C}, \mathrm{O}$ 의 형 태를 찾아 사진을 찍고 facebook에 올리세요. 흥미 로운 사진을 통해 많은 사람들이 관심을 가질 것 입니다.

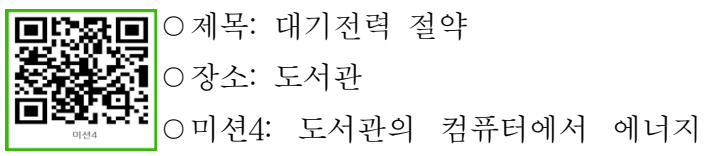
가 낭비되고 있다는 신고가 접수되었습니다. 도서 관 컴퓨터에 '그린터치'프로그램을 설치하여 대기전 력을 절약하고, 수행모습을 facebook에 올리세요.

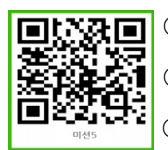

제목: 옥상녹화 ○장소: 제한 없음 미션5: 학교 건물 옥상에서 많은 양의 에너지가 낭비되고 있습니다. 이를 막기 위해 옥상 을 녹화할 수 있는 건물 3 곳을 찾아 사진을 찍고, 인터넷을 이용해 옥상녹화 효과를 3 가지 이상 찾아 facebook에 모두 올리세요.

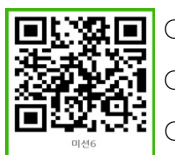

○ 제목: 공중 부양

장소: 제한 없음

미션6: 팀원들과 함께 열심히 미션을 수행하고 있나요? 이번 미션에서는 협동심을 테스트 하겠습니다. 사진을 찍는 홍보실장을 제외한 5명의 팀원이 동시에 공중부양을 한 모습을 찍어 facebook 에 올려주세요. 미션을 완료했나요? 다음 미션을 위 해 인문관 잔디밭에서 $\mathrm{QR}$ 코드를 찾으세요.

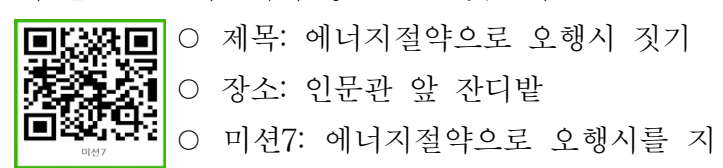


어 facebook에 올리세요. 미션을 완료했나요? 인문 관 앞 잔디밭에 있는 교사를 찾아가세요.

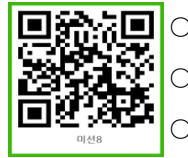

제목: 교사와 함께하는 스피드게임 장소: 인문관 앞 잔디밭

미션8: 이전에 학습한 내용을 바탕으 로 짝을 이루어 스피드 게임을 진행합니다. 제한 시 간 1 분 내에 5 문제 이상 맞출 경우 다음 미션을 위 한 초성힌트를 제공받을 수 있습니다.교사가 있는 인문관 잔디밭으로 와서 팀 구성원 중 2명이 짝을 이루어 스피드 게임을 합니다. 스피드 게임에 대한 내용은 에너지와 관련하여 전 차시에 학습한 내용 을 중점으로 문제가 제시됩니다. 미션을 완료했나 요? 교사에게 다음 미션 $\mathrm{QR}$ 코드를 제공받으세요.

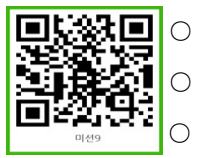

제목: 태양전지 입지조건

장소: 제한없음

미션9: $\mathrm{QR}$ 코드에 제시된 태양전지 의 입지조건에 대한 개념 및 설명을 읽고 이해한 후, 그에 맞는 장소를 찾아 사진을 찍어 facebook 에 올리세요. 태양전지란 태양빛의 에너지를 전기에 너지로 바꾸는 것이며, 태양전지 입지조건을 찾기 위해서는 태양이 항상 잘 들고 그늘이 생기지 않고 반사체를 설치할 공간이 넓고, 주변 건물에 의한 그 림자가 생기지 않는 곳을 찾으세요. 미션을 완료했 나요? 다음 미션을 위해 융합관 건물 1 층으로 가서 $\mathrm{QR}$ 코드를 찾으세요.

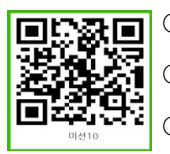

제목: 태양에너지의 이용

장소: 융합관 1 층

미션10: 태양에너지를 이용하는 방법

2 가지를 생각해보고, 환경교육과 교수님에게 자문을 구하고 인터뷰 영상을 facebook에 올리세요. 태양 에너지는 순수한 복사에너지의 형태로 지구에 도달 하며, 자연에서 얻을 수 있고 그 에너지의 양은 매 우 많다. 그러나 지속적인 공급이 불가능하며, 태 양에너지는 확산에너지로, 모든 양의 에너지를 모 으고 변환하고 저장하는 것이 불가능하다. (예: 넓 은 범위에 퍼진 빛을 여러 개의 반사체 거울, 알루 미늄박 등을 이용하여 한곳으로 모은다.) 자! 이제 마지막 미션만이 남았군요. 도서관 1 층 컴퓨터로
가서 $\mathrm{QR}$ 코드를 찾으세요.

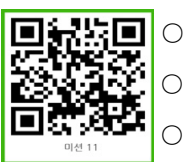

제목: 홍보포스터 제작

장소: 도서관 1 층 컴퓨터

미션11: 우리는 친환경 교원대를 만 들기 위해 10 가지 미션을 수행하였습니다. 이제는 우리가 수행한 미션들을 바탕으로 링크를 참고하여 홍보포스터를 제작하시기 바랍니다. 완성된 포스터 를 기숙사에 하루 동안 붙인 후 가장 많은 스티커 를 받은 팀을 선정할 것입니다. 글로그스터는 한글 입력이 되지 않습니다. 한글을 넣기 위해서는 그림 판에서 한글을 입력하여 이미지로 넣으셔야 합니다.

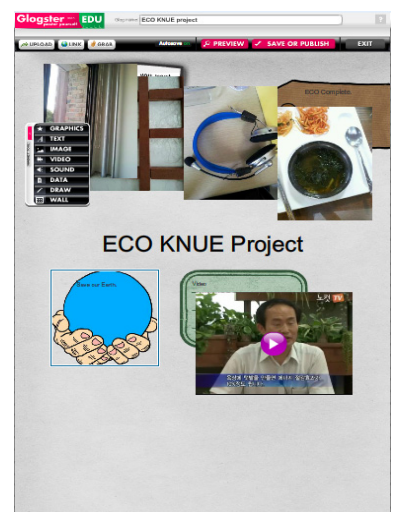

[Fig. 2] Case of Glogster making

\section{4. 결 론}

학습자들의 학습과제에 대한 가치 인식수준을 높여 학습동기를 유발하기 위해서는 실제와 같은 학습활동이 필요하다. 학습자들의 요구를 효과적으 로 반영하여 학습자중심 학습이 되기 위해서는 체 계적인 교수설계 과정을 바탕으로 요구분석과 학습 자 특성분석 이를 토대로 수준에 맞는 학습과제분 석과 설계, 학습자들의 동기를 촉진하는 게임형 교 수설계가 대안이 될 수 있다. 학습자들이 교수-학 습 활동 과정에서 효율적인 학습목표 달성을 위해 서는 개방적이며 동기촉진적인 학습 환경을 만들 필요가 있는 것이다. $\mathrm{QR}$ 코드는 학습자들에게 개방 
적인 학습자 참여 활동 기회를 제공하며 팀간, 팀 내 활동과정에서 협력과정과 상호작용, 의사소통을 통해 지식과 경험을 공유하는 집단지성을 통한 문 제해결을 가능하게 하였다.

무엇보다 교육활동에서 사용되는 $\mathrm{QR}$ 코드와 같 이 소양을 익히는데 많은 시간을 할애하지 않고, 학습활동을 설계하고 활용하는 기회가 된다면 교사 에게는 수업준비에 부담을 덜 수 있고, 학습자들은 기능에 대한 어려움 없이 학습활동 본연의 목적을 달성하는데 많은 시간을 할애하는 기회를 제공할 수 있는 것이다. 추후 체계적인 설계 사례를 바탕 으로 교수설계 과정에서 소요되는 시간에 대해 초 보 교수자의 설계과정을 질적으로 분석하고 학습자 들의 학업성취나 문제해결 능력, 전이능력을 살피 는 연구가 진행 되어야 할 것이다. 또한 평가 절차 를 위해 개발된 $\mathrm{QR}$ 코드를 활용하여 활동과정에 내재된 다양한 변인의 성과와 문제를 확인하는 연 구도 진행될 필요가 있다.

\section{REFERENCES}

[1] von Glasersfeld, "Quesions and answers about radical constructivism". In K. Tobin (Ed.), The practice of constructivism in science education. pp23-38. Hillsdale, NJ: Lawrence Erlbaum Associates, 1993.

[2] Duran, T., \& Gauvain, M, "The role of age versus expertise in peer collaboration during joint planning", Journal of Experimental Child Psychology, Vol 55, pp227-242, 1993.

[3] Kim, S. J, "The Implication of Media Environment Change Caused by the Emergence of Web 2.0", Korea Information Science Society review, Vol 25, Num 10, pp12-15, 2007.

[4] Park, H. S,, "Case Study and Development of Quest-Based Learning Using QR Code", Journal of Korea Game Society, Vol 11, Num 5, pp79-88, 2011.

[5] Libin, A.V. \& E. Libin, "Person-Robot Interactions. From the Robopsychologists'
Point of View: The Robotic", Psychology and Robotherapy Approach, Proceeding of the IEEE, Vol 92, Num 15, pp1-15, 2004.

[6] Levy, D, "Love and Sex with Robots: The Evolution of Human-Robot Relationships", Publisher: Harper Perennial, 2008.

[7] Plowman L. \& Stephen C, "A 'benign addition'? Research on ICT and pre-school children", Journal of Computer-Assisted Learning, Vol 19, Num 2, pp149-164, 2003.

[8] Wolfinger, D. M., \& Stockard, J. W, “ Elementary methods: An integrated curriculum. New York: Longman, 1997.

[9] Yang, M. K, "Exploration of Methodological Principle and Meaning of Curriculum Integration", Journal of Educational Research, Vol 35, Num 4, pp111-132, 1997.

[10] Spanos, G. "On the integration of language and content instruction”, In R. B. Kaplan (Ed.), Annual Review of Applied Linguistics 10 (pp.227-240). New York: Cambridge University Press, 1990.

[11] Jacobs, H. H, "The growing need for interdisciplinary curriculum content", In H. H. Jacobs (Ed.), Interdisciplinary curriculum: Design and implementation(pp.1-11). Alexandria, VA: Association for Supervision and Curriculum Development, 1990.

[12] Ackerman, D. B, "Intellectual and practical criteria for successful curriculum integration", In H. H. Jacobs (Ed.), Interdisciplinary curriculum: Design and implementation(pp. 1-11). Alexandria, VA: Association for Supervision \& Curriculum Development, 1990.

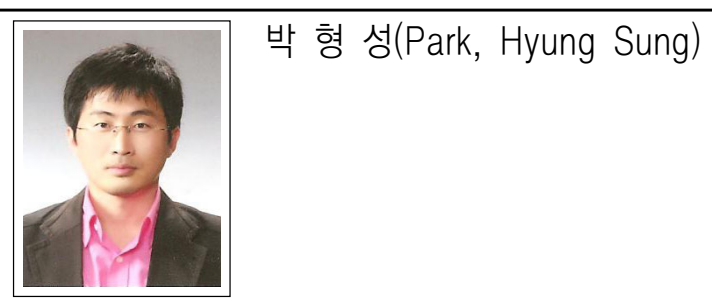

한국교원대학교 겸임교수/ 경기대학교 강사

관심분야 : 교육용게임, $\mathrm{e}^{-}$러닝, 게임기반학습 
$-\mathrm{QR}$ 코드를 활용한 주제중심 통합학습 설계 사례연구- 